\title{
ON THE MULTIPLICITY OF REES ALGEBRAS OF GOOD FILTRATIONS
}

\author{
Duong Quoc VIET and Le Van DINH \\ (Received 17 December 2010 and revised 6 December 2011)
}

\begin{abstract}
Let $(A, \mathfrak{m})$ be a Noetherian local ring. Let $\mathcal{F}=\left\{I_{n}\right\}_{n \geq 0}$ be a good filtration of ideals in $A$. Denote by $R(\mathcal{F})=\bigoplus_{n \geq 0} I_{n} t^{n}$ the Rees algebra of $\mathcal{F}$. In this paper we investigate the mixed multiplicities of $\mathcal{F}$ and the relationship between the multiplicity of $R(\mathcal{F})$ and the mixed multiplicities of $\mathcal{F}$. As an application, we obtain several multiplicity formulas that express the multiplicity of $R(\mathcal{F})$ as a sum of Hilbert-Samuel multiplicities of some quotient rings of $A$.
\end{abstract}

\section{Introduction}

Let $(A, \mathfrak{m})$ be a Noetherian local ring of Krull dimension $d=\operatorname{dim} A>0$ with infinite residue field $k=A / \mathfrak{m}$. Let $\mathcal{F}=\left\{I_{n}\right\}_{n \geq 0}$ be a good filtration of ideals in $A$. Define

$$
R(\mathcal{F})=\bigoplus_{n \geq 0} I_{n} t^{n}
$$

to be the Rees algebra of $\mathcal{F}$. In the case when $\mathcal{F}=\left\{I^{n}\right\}_{n \geq 0}$ is an $I$-adic filtration, $R(\mathcal{F})$ is called the Rees algebra of $I$ and denoted by $R(I)$.

The multiplicity of the Rees algebra of an ideal has been studied in many works (see e.g. $[3,4,11-13]$. Verma in [11] expressed the Hilbert-Samuel multiplicity of $R(I)$ as the sum of all the mixed multiplicities of $I$. Viet in [12] described the multiplicity of $R(I)$ as a sum of Hilbert-Samuel multiplicities of some quotient rings of $A$.

The aim of this paper is to give generalizations of the above-mentioned results in [12] to the case of the Rees algebra of a good filtration. We first define mixed multiplicities of a good filtration (see Section 2). Next we interpret each mixed multiplicity of a good filtration as the Hilbert-Samuel multiplicity of a quotient ring of $A$ (see Theorem 3.7). Then we express the multiplicity of $R(\mathcal{F})$ as the sum of all the mixed multiplicities of $\mathcal{F}$ (see Proposition 4.2). As a consequence of Theorem 3.7 and Proposition 4.2, we obtain an explicit formula for the Hilbert-Samuel multiplicity of the Rees algebra of a good filtration that expresses the multiplicity of $R(\mathcal{F})$ as a sum of Hilbert-Samuel multiplicities of some quotient rings of $A$ (see Theorem 4.6). In the case when $\mathcal{F}$ is an equimultiple filtration, we obtain a multiplicity formula for $R(\mathcal{F})$ (see Corollary 4.7) which is a generalization of the multiplicity formula for the Rees algebra of an ideal in [13, Theorem 3.2]. Our main results not only cover several

2010 Mathematics Subject Classification: Primary 13H15; Secondary 13A30, 13D40, 14 C17.

Keywords: multiplicity; filtration; Rees algebra; mixed multiplicity.

(C) 2012 Faculty of Mathematics, Kyushu University 
earlier results, but also yield interesting consequences (see, e.g., Remark 2.3, Lemma 3.3, Remark 3.8, Lemma 4.1, Remark 4.4, Corollary 4.5).

This paper is divided into four sections. Section 2 is devoted to the discussion of mixed multiplicities of a good filtration. In Section 3 we investigate the relationship between mixed multiplicities of a good filtration and Hilbert-Samuel multiplicities via (FC)-sequences (see Definition 3.1). Finally, in Section 4 we establish the multiplicity formula for $R(\mathcal{F})$ which expresses the multiplicity of $R(\mathcal{F})$ as a sum of Hilbert-Samuel multiplicities of some quotient rings of $A$.

\section{Mixed multiplicities of a good filtration}

In this section, we define mixed multiplicities of a good filtration. We first recall some notions that will be used in this paper.

A filtration $\mathcal{F}=\left\{I_{n}\right\}_{n \geq 0}$ of ideals in $A$ is a chain of ideals $I_{n}$ such that $I_{0}=A, I_{1}$ is a proper ideal of $A, I_{n+1} \subseteq I_{n}$, and $I_{m} I_{n} \subseteq I_{m+n}$ for all $m, n \geq 0$. Let $I$ be an ideal of $A$. $\mathcal{F}$ is called an $I$-good filtration if $I I_{n} \subseteq I_{n+1}$ for all $n \geq 0$ and $I_{n+1}=I I_{n}$ for all large $n$. In this case, $I \subseteq I_{1} . \mathcal{F}$ is called a good filtration if it is an $I$-good filtration for some ideal $I$ of $A$. It is easily seen that $\mathcal{F}$ is a good filtration if and only if $\mathcal{F}$ is an $I_{1}$-good filtration. There are numerous examples of good filtrations; for instance, for an ideal $I$ of $A,\left\{\widetilde{I^{n}}\right\}_{n \geq 0}$ is a good filtration, where $\widetilde{I}^{n}$ is the Ratliff-Rush closure of $I^{n}$ (see [6, Theorem 2.1]). Moreover, if $A$ is analytically unramified containing a field, then $\left\{{\overline{I^{n}}}_{n \geq 0}\right.$ and $\left\{\left(I^{n}\right)^{*}\right\}_{n \geq 0}$ are good filtrations; here $\overline{I^{n}}$ and $\left(I^{n}\right)^{*}$ denote the integer closure and tight closure of $I^{n}$, respectively (see $\left.[2,7]\right)$.

From now on, let $\mathcal{F}=\left\{I_{n}\right\}_{n \geq 0}$ be a good filtration of ideals in $A$. We call $\mathcal{F}$ a nilpotent filtration if $I_{n}=0$ for all large $n$. Observe that $\mathcal{F}$ is a nilpotent filtration if and only if $I_{1}$ is nilpotent. Let $J$ be an $\mathfrak{m}$-primary ideal of $A$. Denote by $F_{J}(\mathcal{F})=\bigoplus_{n \geq 0}\left(I_{n} / J I_{n}\right) t^{n}$ the fiber cone of $\mathcal{F}$ with respect to $J$. If $\mathcal{F}=\left\{I^{n}\right\}_{n \geq 0}$ is an $I$-adic filtration, we denote $F_{J}(\mathcal{F})$ by $F_{J}(I)$ and call it the fiber cone of $I$ with respect to $J$. The number $\ell(I)=\operatorname{dim} F_{\mathfrak{m}}(I)$ is called the analytic spread of $I$. Since $J$ is an m-primary ideal of $A$, in $R(\mathcal{F})$ we have $\sqrt{J R(\mathcal{F})}=\sqrt{\mathfrak{m} R(\mathcal{F})}$. Hence $\operatorname{dim} F_{J}(\mathcal{F})=\operatorname{dim} F_{\mathfrak{m}}(\mathcal{F})=\ell\left(I_{1}\right)$. It is well known that ht $I \leq \ell(I) \leq \operatorname{dim} A$, where ht $I$ is the height of $I$ [5]. In the case when ht $I=\ell(I), I$ is called an equimultiple ideal. If $I_{1}$ is equimultiple, we call $\mathcal{F}$ an equimultiple filtration. Set $\mathcal{F} / I=\left\{I_{n}(A / I)\right\}_{n \geq 0}$ and

$$
F_{J}(\mathcal{F} / I)=\bigoplus_{n \geq 0}\left[\left(I_{n}+I\right) /\left(J I_{n}+I\right)\right] t^{n}
$$

for any ideal $I$ of $A$.

Note 1. Put $\ell=\ell\left(I_{1}\right)$. Then $\ell_{A}\left(I_{n} / J I_{n}\right)$ is a polynomial of degree $\ell-1$ for all large $n$ by [16, Remark 2.1(vii)].

We now turn to the definition of mixed multiplicities of a good filtration. Let $\mathcal{F}=\left\{I_{n}\right\}_{n \geq 0}$ be a good filtration of ideals in $A$ and let $J$ be an $m$-primary ideal of $A$. Consider the following bigraded algebras:

$$
T(J, \mathcal{F})=\bigoplus_{m, n \geq 0} \frac{J^{m} I_{n}}{J^{m+1} I_{n}} t_{1}^{m} t_{2}^{n} \quad \text { and } \quad T\left(J, I_{1}\right)=\bigoplus_{m, n \geq 0} \frac{J^{m} I_{1}^{n}}{J^{m+1} I_{1}^{n}} t_{1}^{m} t_{2}^{n},
$$


where $t_{1}$ and $t_{2}$ are indeterminates. It is easily seen that $T\left(J, I_{1}\right)$ is a standard bigraded algebra. Set $T_{j}=\bigoplus_{m \geq 0, n \geq j}\left(J^{m} I_{n} / J^{m+1} I_{n}\right) t_{1}^{m} t_{2}^{n}$ for $j \geq 0$. Then $T_{j}$ has a natural bigraded $T\left(J, I_{1}\right)$-module structure given by

$$
\left(a+J^{m+1} I_{1}^{n}\right)\left(x+J^{k+1} I_{l}\right)=a x+J^{m+k+1} I_{n+l}
$$

for $a \in J^{m} I_{1}^{n}$ and $x \in J^{k} I_{l}$. Since $\mathcal{F}$ is a good filtration, we have

$$
T_{j}=\bigoplus_{m \geq 0, n \geq j} \frac{J^{m} I_{n}}{J^{m+1} I_{n}} t_{1}^{m} t_{2}^{n}=T\left(J, I_{1}\right)\left(I_{j} / J I_{j}\right) t_{2}^{j}
$$

for all large $j$. Hence $T_{j}$ is a finitely generated $T\left(J, I_{1}\right)$-module. Consequently, the Bhattacharya function $B(m, n)=\ell_{A}\left(J^{m} I_{n} / J^{m+1} I_{n}\right)$ is a polynomial for all large $m, n$. Denote by $P(m, n)$ the polynomial of the function $B(m, n)$. Then $P(m, n)$ is called the Bhattacharya polynomial of $B(m, n)$ [1].

Set $\mathfrak{a}: \mathfrak{b}^{\infty}=\bigcup_{n \geq 0}\left(\mathfrak{a}: \mathfrak{b}^{n}\right)$. We need the following lemma.

LEMMA 2.1. Let $\mathcal{F}=\left\{I_{n}\right\}_{n \geq 0}$ be a non-nilpotent good filtration of ideals in A. Set $A^{*}=$ $A / 0: I_{1}^{\infty}$ and $q=\operatorname{dim} A^{*}$. Then:

(i) $\left(0: I_{1}^{\infty}\right) \cap I_{n}=0$ for all large $n$;

(ii) grade $I_{1} A^{*} \geq 1$ and $q \geq 1$.

Proof. Since $\mathcal{F}$ is a good filtration, there exists a non-negative integer $u$ such that $I_{n}=I_{1}^{n-u} I_{u}$ for all large $n$. By the Artin-Rees lemma, there exists a non-negative integer $v$ such that for all $n-u \geq v$,

$$
\left(0: I_{1}^{\infty}\right) \cap I_{n} \subseteq\left(0: I_{1}^{\infty}\right) \cap I_{1}^{n-u}=\left(\left(0: I_{1}^{\infty}\right) \cap I_{1}^{v}\right) I_{1}^{n-u-v} \subseteq\left(0: I_{1}^{\infty}\right) I_{1}^{n-u-v} .
$$

Hence, since $\left(0: I_{1}^{\infty}\right) I_{1}^{n-u-v}=0$ for $n$ large enough, it follows that $\left(0: I_{1}^{\infty}\right) \cap I_{n}=0$ for all large $n$. We get (i).

To proof (ii), set $I_{1}^{*}=I_{1} A^{*}$. Note that $I_{1}$ is non-nilpotent, $A^{*} \neq 0$. Since $\left(0: I_{1}^{\infty}\right): I_{1}=$ $0: I_{1}^{\infty}$, then $0_{A^{*}}: I_{1}^{*}=0_{A^{*}}$. This implies that grade $I_{1}^{*} \geq 1$, and hence $q=\operatorname{dim} A^{*} \geq 1$.

Proposition 2.2. Let $J$ be an $\mathfrak{m}$-primary ideal of $A$ and let $\mathcal{F}=\left\{I_{n}\right\}_{n \geq 0}$ be a nonnilpotent good filtration of ideals in A. Set $q=\operatorname{dim} A / 0: I_{1}^{\infty}$. Then the Bhattacharya function $\ell_{A}\left(J^{m} I_{n} / J^{m+1} I_{n}\right)$ is a polynomial of degree $q-1$ for all large $m, n$.

Proof. Set $\mathcal{E}=\left\{J^{n} I_{n}\right\}_{n \geq 0}$. Since $\mathcal{F}$ is a good filtration, $\mathcal{E}$ is also a good filtration. Let $P(m, n)$ be the Bhattacharya polynomial of the function $\ell_{A}\left(J^{m} I_{n} / J^{m+1} I_{n}\right)$. By Note $1, \operatorname{deg} P(n, n)=\operatorname{dim} F_{J}(\mathcal{E})-1=\ell\left(J I_{1}\right)-1$. On the other hand, $\operatorname{deg} P(m, n)=$ $\operatorname{deg} P(n, n)$, since the highest coefficients of $P(m, n)$ are non-negative numbers, not all zero. Hence $\operatorname{deg} P(m, n)=\ell\left(J I_{1}\right)-1$. Denote by $Q(m, n)$ the polynomial of the function $\ell_{A}\left(J^{m} I_{1}^{n} / J^{m+1} I_{1}^{n}\right)$. By [12, Proposition 3.1], $\operatorname{deg} Q(m, n)=q-1$. By Note 1, $\operatorname{deg} Q(n, n)=\ell\left(J I_{1}\right)-1$. It is easily seen that $\operatorname{deg} Q(m, n)=\operatorname{deg} Q(n, n)$. Hence we get $\operatorname{deg} P(m, n)=q-1$.

Remark 2.3. From the proof of Proposition 2.2, it follows that if $J$ is an $\mathfrak{m}$-primary ideal and $I$ is an arbitrary ideal then $\ell(J I)=\operatorname{dim} A / 0: I^{\infty}$. Note that in the case ht $I>0$, $\ell(J I)=\operatorname{dim} A$. This fact was shown by Viet [15, Theorem 5.2(ii)]. 
Write the Bhattacharya polynomial of $B(m, n)=\ell_{A}\left(J^{m} I_{n} / J^{m+1} I_{n}\right)$ in the form

$$
\begin{aligned}
P(m, n)= & \sum_{d_{0}+d_{1}=q-1} e_{A}\left(J^{\left[d_{0}+1\right]}, \mathcal{F}^{\left[d_{1}\right]}\right)\left(\begin{array}{c}
m+d_{0} \\
d_{0}
\end{array}\right)\left(\begin{array}{c}
n+d_{1} \\
d_{1}
\end{array}\right) \\
& +\sum_{i+j<q-1} e_{A}\left(J^{[i+1]}, \mathcal{F}^{[j]}\right)\left(\begin{array}{c}
m+i \\
i
\end{array}\right)\left(\begin{array}{c}
n+j \\
j
\end{array}\right) ;
\end{aligned}
$$

then $e_{A}\left(J^{\left[d_{0}+1\right]}, \mathcal{F}^{\left[d_{1}\right]}\right)$ are non-negative integers not all zero. We call $e_{A}\left(J^{\left[d_{0}+1\right]}, \mathcal{F}^{\left[d_{1}\right]}\right)$ the mixed multiplicity of the good filtration $\mathcal{F}$ with respect to $J$ of the type $\left(d_{0}+1, d_{1}\right)$.

\section{Computing mixed multiplicities of a filtration}

The relationship between mixed multiplicities of ideals and Hilbert-Samuel multiplicity was studied first for m-primary ideals by Risler and Teissier (1973) [9], Rees (1984) [8] and then for arbitrary ideals by Viet (2000) [12]. In this section we develop the method of Viet in [12] to interpret mixed multiplicities of a good filtration as the Hilbert-Samuel multiplicity. We recall here the concept of (FC)-sequences of good filtrations that were introduced in [16]. This is a kind of variant of superficial sequences.

Definition 3.1. Let $I$ be an ideal of $A$ and let $\mathcal{F}=\left\{I_{n}\right\}_{n \geq 0}$ be a non-nilpotent good filtration of ideals in $A$. An element $x \in I_{1}$ is called an $(F C)$-element with respect to $(I, \mathcal{F})$ if the following conditions are satisfied:

(FC1) $I^{m} I_{n} \cap(x)=x I^{m} I_{n-1}$ for all $m \geq 0$ and all large $n$;

(FC2) $x$ is a filter-regular element with respect to $I_{1}$, i.e., $0: x \subseteq 0: I_{1}^{\infty}$;

(FC3) $\operatorname{dim}\left[A /(x): I_{1}^{\infty}\right]=\operatorname{dim}\left[A / 0: I_{1}^{\infty}\right]-1$.

We call $x$ a weak- $(F C)$-element with respect to $(I, \mathcal{F})$ if $x$ satisfies conditions (FC1) and (FC2).

Let $x_{1}, \ldots, x_{s}$ be a sequence in $I_{1}$. For each $i=0,1, \ldots, s-1$, set $A_{i}=$ $A /\left(x_{1}, \ldots, x_{i}\right), \bar{x}_{i+1}$ is the image of $x_{i+1}$ in $A_{i}$, and $\mathcal{F}_{i}=\left\{I_{n} A_{i}\right\}_{n \geq 0}$. Then $x_{1}, \ldots, x_{s}$ is called an $(F C)$-sequence (respectively, a weak-(FC)-sequence) with respect to $(I, \mathcal{F})$ if $\bar{x}_{i+1}$ is an (FC)-element (respectively, a weak-(FC)-element) with respect to $\left(I A_{i}, \mathcal{F}_{i}\right)$ for each $i=0,1, \ldots, s-1$.

A weak-(FC)-sequence $x_{1}, \ldots, x_{s}$ in $I_{1}$ with respect to $(I, \mathcal{F})$ is called a maximal weak(FC)-sequence if $\mathcal{F}_{s-1}$ is a non-nilpotent filtration of $A_{s-1}$ and $\mathcal{F}_{s}$ is a nilpotent filtration of $A_{s}$.

The existence of weak-(FC)-sequences of good filtrations was shown in [16].

Lemma 3.2. [16, Proposition 2.3] Let I be an ideal of $A$ and let $\mathcal{F}=\left\{I_{n}\right\}_{n \geq 0}$ be a nonnilpotent good filtration of ideals in A. Then there exists a weak-(FC)-element in $I_{1}$ with respect to $(I, \mathcal{F})$.

Basic facts about weak-(FC)-sequences of good filtrations were pointed out in [16]. In the following we also give some properties of these sequences.

LEMMA 3.3. Let I be an ideal of $A$ and let $\mathcal{F}=\left\{I_{n}\right\}_{n \geq 0}$ be a non-nilpotent good filtration of ideals in A. Suppose that $x$ is a weak- $(F C)$-element in $I_{1}$ with respect to $(I, \mathcal{F})$, and that 
$\mathfrak{b} \subseteq \mathfrak{a}$ are ideals in A. Then we have the following isomorphisms of A-modules for all large $n:$

$$
\mathfrak{a} I_{n} / \mathfrak{b} I_{n} \simeq x \mathfrak{a} I_{n} / x \mathfrak{b} I_{n} .
$$

Proof. It is enough to show that $\mathfrak{a} I_{n} \simeq x \mathfrak{a} I_{n}$ for all large $n$. It will follow that

$$
\mathfrak{a} I_{n} / \mathfrak{b} I_{n} \simeq x \mathfrak{a} I_{n} / x \mathfrak{b} I_{n}
$$

for all large $n$. For each $n$, consider the map

$$
\begin{aligned}
\lambda_{n}: \mathfrak{a} I_{n} & \longrightarrow x \mathfrak{a} I_{n} \\
y & \longmapsto x y .
\end{aligned}
$$

Then $\lambda_{n}$ is surjective and $\operatorname{Ker} \lambda_{n}=\mathfrak{a} I_{n} \cap(0: x)$. Since $x$ is a weak-(FC)-element with respect to $(I, \mathcal{F})$, it follows from Lemma 2.1(i) that

$$
\operatorname{Ker} \lambda_{n}=\mathfrak{a} I_{n} \cap(0: x) \subseteq I_{n} \cap\left(0: I_{1}^{\infty}\right)=0
$$

for all large $n$. Hence $\lambda_{n}$ are isomorphisms; that is, $\mathfrak{a} I_{n} \simeq x \mathfrak{a} I_{n}$ for all large $n$.

The length of maximal weak-(FC)-sequences of ideals was studied by Viet in [13] and [14]. In the case of good filtrations, we recall the following result from [16].

LEMma 3.4. [16, Proposition 2.4(iii)] Let $J$ be an $\mathfrak{m}$-primary ideal of $A$ and let $\mathcal{F}=\left\{I_{n}\right\}_{n \geq 0}$ be a good filtration of ideals in A. Then the length of maximal weak-(FC)-sequences in $I_{1}$ with respect to $(J, \mathcal{F})$ is $\ell\left(I_{1}\right)$.

Note 2. Let $x \in I_{1}$ be a weak-(FC)-element with respect to $(J, \mathcal{F})$. Set $\bar{A}=A /(x), \bar{J}=J \bar{A}$, and $\bar{I}_{n}=I_{n} \bar{A}$ for all $n \geq 0$. Then $\mathcal{F} /(x)=\left\{\bar{I}_{n}\right\}_{n \geq 0}$ and for all large $n$, we have

$$
\begin{aligned}
\ell_{\bar{A}}\left(\frac{\bar{I}_{n}}{\bar{J} \bar{I}_{n}}\right) & =\ell_{A}\left(\frac{I_{n}+(x)}{J I_{n}+(x)}\right) \\
& =\ell_{A}\left(\frac{I_{n}}{J I_{n}+(x) \cap I_{n}}\right) \\
& =\ell_{A}\left(\frac{I_{n}}{J I_{n}}\right)-\ell_{A}\left(\frac{J I_{n}+(x) \cap I_{n}}{J I_{n}}\right) \\
& =\ell_{A}\left(\frac{I_{n}}{J I_{n}}\right)-\ell_{A}\left(\frac{(x) \cap I_{n}}{(x) \cap J I_{n}}\right) \\
& =\ell_{A}\left(\frac{I_{n}}{J I_{n}}\right)-\ell_{A}\left(\frac{x I_{n-1}}{x J I_{n-1}}\right) \quad(\text { since } x \text { satisfies condition (FC1)) } \\
& =\ell_{A}\left(\frac{I_{n}}{J I_{n}}\right)-\ell_{A}\left(\frac{I_{n-1}}{J I_{n-1}}\right) \quad \text { (by Lemma 3.3). }
\end{aligned}
$$

Hence

$$
\ell_{\bar{A}}\left(\frac{\bar{I}_{n}}{\bar{J} \bar{I}_{n}}\right)=\ell_{A}\left(\frac{I_{n}}{J I_{n}}\right)-\ell_{A}\left(\frac{I_{n-1}}{J I_{n-1}}\right) .
$$

LEMMA 3.5. Let $J$ be an $\mathfrak{m}$-primary ideal of $A$ and let $\mathcal{F}=\left\{I_{n}\right\}_{n \geq 0}$ be a non-nilpotent good filtration of ideals in A. Set $A^{*}=A / 0: I_{1}^{\infty}$ and $q=\operatorname{dim} A^{*}$. Then: 
(i) $e\left(J, I_{n}\right)=e\left(J, A^{*}\right)$ for all large $n$;

(ii) $e_{A}\left(J^{[q]}, \mathcal{F}^{[0]}\right)=e\left(J, A^{*}\right)$. Moreover, if ht $I_{1}>0$ then $e_{A}\left(J^{[q]}, \mathcal{F}^{[0]}\right)=e(J, A)$.

Proof. We have $\left(0: I_{1}^{\infty}\right) \cap I_{n}=0$ for all large $n$ by Lemma 2.1(i). It follows that the map

$$
\begin{aligned}
& I_{n} \longrightarrow A / 0: I_{1}^{\infty} \\
& x \longmapsto x+0: I_{1}^{\infty}
\end{aligned}
$$

is an injection for $n$ large enough. Consequently, we get the following exact sequences

$$
0 \longrightarrow I_{n} \longrightarrow A^{*} \longrightarrow A^{*} / I_{n} A^{*} \longrightarrow 0
$$

for all large $n$. Note that grade $I_{1} A^{*} \geq 1$ by Lemma 2.1(ii). Therefore, $\operatorname{ht}\left(I_{n} A^{*}\right)=\operatorname{ht}\left(I_{1} A^{*}\right)>$ 0 for all $n \geq 1$ since $I_{1}^{n} \subseteq I_{n} \subseteq I_{1}$. This implies that $\operatorname{dim}\left(A^{*} / I_{n} A^{*}\right)<\operatorname{dim} A^{*}$ for all $n \geq 1$. Now the above exact sequences give $e\left(J, I_{n}\right)=e\left(J, A^{*}\right)$ for all large $n$. We obtain (i). Let us prove (ii). It is easily seen that

$$
\begin{aligned}
e_{A}\left(J^{[q]}, \mathcal{F}^{[0]}\right) & =\lim _{m \rightarrow \infty} \frac{(q-1) !}{m^{q-1}} \ell_{A}\left(J^{m} I_{n} / J^{m+1} I_{n}\right) \text { for all large } n \\
& =e\left(J, I_{n}\right) \quad \text { for all large } n .
\end{aligned}
$$

It now follows from (i) that $e_{A}\left(J^{[q]}, \mathcal{F}^{[0]}\right)=e\left(J, A^{*}\right)$. If ht $I_{1}>0$, then since ht $I_{n}=$ ht $I_{1}>0$, it follows that $\operatorname{dim}\left(A / I_{n}\right)<\operatorname{dim} A$ for all $n \geq 1$. Hence the exact sequence

$$
0 \longrightarrow I_{n} \longrightarrow A \longrightarrow A / I_{n} \longrightarrow 0
$$

yields $e\left(J, I_{n}\right)=e(J, A)$, which together with (1) implies that $e_{A}\left(J^{[q]}, \mathcal{F}^{[0]}\right)=e(J, A)$.

PROPOSITION 3.6. Let $J$ be an $\mathfrak{m}$-primary ideal of $A$ and let $\mathcal{F}=\left\{I_{n}\right\}_{n \geq 0}$ be a non-nilpotent good filtration of ideals in A. Set $A^{*}=A / 0: I_{1}^{\infty}, q=\operatorname{dim} A^{*}$, and $\ell=\ell\left(I_{1}\right)$. Let $d_{0}, d_{1}$ be non-negative integers such that $d_{0}+d_{1}=q-1$. Then the following statements hold.

(i) If $e_{A}\left(J^{\left[d_{0}+1\right]}, \mathcal{F}^{\left[d_{1}\right]}\right) \neq 0$ and $d_{1}>0$, then there exists an $(F C)$-element $x \in I_{1}$ with respect to $(J, \mathcal{F})$.

(ii) If $x \in I_{1}$ is an (FC)-element with respect to $(J, \mathcal{F})$ and $d_{1}>0$, then

$$
e_{A}\left(J^{\left[d_{0}+1\right]}, \mathcal{F}^{\left[d_{1}\right]}\right)=e_{\bar{A}}\left(\bar{J}^{\left[d_{0}+1\right]}, \overline{\mathcal{F}}^{\left[d_{1}-1\right]}\right),
$$

where $\bar{A}=A /(x), \bar{J}=J \bar{A}$, and $\overline{\mathcal{F}}=\left\{I_{n} \bar{A}\right\}_{n \geq 0}$.

(iii) If $e_{A}\left(J^{\left[d_{0}+1\right]}, \mathcal{F}^{\left[d_{1}\right]}\right) \neq 0$ and $x_{1}, \ldots, x_{d_{1}} \in I_{1}$ is a weak-(FC)-sequence with respect to $(J, \mathcal{F})$, then $x_{1}, \ldots, x_{d_{1}}$ is an $(F C)$-sequence with respect to $(J, \mathcal{F})$.

(iv) If $x_{1}, \ldots, x_{s} \in I_{1}$ is a weak-(FC)-sequence with respect to $(J, \mathcal{F})$, then

$$
\operatorname{dim} A /\left[\left(x_{1}, \ldots, x_{s}\right): I_{1}^{\infty}\right] \leq q-s,
$$

with equality if and only if $x_{1}, \ldots, x_{s}$ is an $(F C)$-sequence with respect to $(J, \mathcal{F})$.

(v) Let $x_{1}, \ldots, x_{\ell}$ be a maximal weak- $(F C)$-sequence in $I_{1}$ with respect to $(J, \mathcal{F})$. If ht $I_{1}=h>0$, then $x_{1}, \ldots, x_{h-1}$ is an $(F C)$-sequence with respect to $(J, \mathcal{F})$. 
Proof. (i) By Lemma 3.2, there exists a weak-(FC)-element $x \in I_{1}$ with respect to $(J, \mathcal{F})$. Set $\bar{A}=A /(x), \bar{J}=J \bar{A}$, and $\bar{I}_{n}=I_{n} \bar{A}$ for all $n \geq 0$. By Note 2 , we have

$$
\ell_{\bar{A}}\left(\frac{\bar{J}^{m} \bar{I}_{n}}{\bar{J}^{m+1} \bar{I}_{n}}\right)=\ell_{A}\left(\frac{J^{m} I_{n}}{J^{m+1} I_{n}}\right)-\ell_{A}\left(\frac{J^{m} I_{n-1}}{J^{m+1} I_{n-1}}\right)
$$

for all large $m, n$. Since $e_{A}\left(J^{\left[d_{0}+1\right]}, \mathcal{F}^{\left[d_{1}\right]}\right) \neq 0$ and $d_{1}>0$, we obtain from (2) that $\ell_{\bar{A}}\left(\bar{J}^{m} \bar{I}_{n} / \bar{J}^{m+1} \bar{I}_{n}\right)$ is a polynomial of degree $q-2$ for all large $m, n$. On the other hand, by Proposition 2.2, the degree of this polynomial is $\operatorname{dim} A /\left[(x): I_{1}^{\infty}\right]-1$. Therefore,

$$
\operatorname{dim}\left[A /(x): I_{1}^{\infty}\right]=q-1=\operatorname{dim}\left[A / 0: I_{1}^{\infty}\right]-1 .
$$

Hence $x$ is an (FC)-element with respect to $(J, \mathcal{F})$.

(ii) Denote by $P(m, n)$ and $\bar{P}(m, n)$ the Bhattacharya polynomials of $\mathcal{F}$ and $\overline{\mathcal{F}}$, respectively. By (2), we have

$$
\bar{P}(m, n)=P(m, n)-P(m, n-1)
$$

for all large $m, n$. By Proposition 2.2, $P(m, n)$ has degree $q-1$, while the degree of $\bar{P}(m, n)$ is $\operatorname{dim} \bar{A} / \overline{0}: \bar{I}_{1}^{\infty}-1$. Since $x$ is an (FC)-element with respect to $(J, \mathcal{F})$, we have

$$
\operatorname{dim} \frac{\bar{A}}{\overline{0}: \bar{I}_{1}^{\infty}}=\operatorname{dim} \frac{A}{(x): I_{1}^{\infty}}=\operatorname{dim} \frac{A}{0: I_{1}^{\infty}}-1=q-1 .
$$

Therefore, $\operatorname{deg} \bar{P}(m, n)=\operatorname{deg} P(m, n)-1$. It now follows from (3) that

$$
e_{A}\left(J^{\left[d_{0}+1\right]}, \mathcal{F}^{\left[d_{1}\right]}\right)=e_{\bar{A}}\left(\bar{J}^{\left[d_{0}+1\right]}, \overline{\mathcal{F}}^{\left[d_{1}-1\right]}\right) .
$$

(iii) We prove by induction on $d_{1}$. When $d_{1}=1$, the result was proved in (i). Assume that the result holds for $d_{1}-1 \geq 1$. Then from the proof of (i) it follows that $x_{1}$ is an (FC)-element with respect to $(J, \mathcal{F})$. Hence by (ii), we have

$$
e_{\bar{A}}\left(\bar{J}^{\left[d_{0}+1\right]}, \overline{\mathcal{F}}^{\left[d_{1}-1\right]}\right)=e_{A}\left(J^{\left[d_{0}+1\right]}, \mathcal{F}^{\left[d_{1}\right]}\right) \neq 0,
$$

where $\bar{A}=A /\left(x_{1}\right), \bar{J}=J \bar{A}$, and $\overline{\mathcal{F}}=\left\{I_{n} \bar{A}\right\}_{n \geq 0}$. Denote by $\bar{x}_{2}, \ldots, \bar{x}_{d_{1}}$ the images of $x_{2}, \ldots, x_{d_{1}}$ in $\bar{A}$, respectively. Then $\bar{x}_{2}, \ldots, \bar{x}_{d_{1}}$ is a weak-(FC)-sequence with respect to $(\bar{J}, \overline{\mathcal{F}})$. By the induction hypothesis, $\bar{x}_{2}, \ldots, \bar{x}_{d_{1}}$ is an (FC)-sequence with respect to $(\bar{J}, \overline{\mathcal{F}})$. So $x_{1}, \ldots, x_{d_{1}}$ is an (FC)-sequence with respect to $(J, \mathcal{F})$. We get (iii).

(iv) Assume that $x \in I_{1}$ is a weak-(FC)-element with respect to $(J, \mathcal{F})$. Set $\bar{A}=A /(x), \bar{J}=J \bar{A}$, and $\overline{\mathcal{F}}=\left\{I_{n} \bar{A}\right\}_{n \geq 0}$. Since $\operatorname{deg} P(m, n)=q-1$ and $\operatorname{deg} \bar{P}(m, n)=$ $\operatorname{dim} A /\left[(x): I_{1}^{\infty}\right]-1$, by (3) we have $\operatorname{dim} A /\left[(x): I_{1}^{\infty}\right] \leq q-1$. By definition, the equality holds in the above inequality if and only if $x$ is an (FC)-element with respect to $(J, \mathcal{F})$. The claim now follows by induction.

(v) By (iv), we have $\operatorname{dim} A /\left[\left(x_{1}, \ldots, x_{h-1}\right): I_{1}^{\infty}\right] \leq q-(h-1)$. Set

$$
\bar{A}=A /\left(x_{1}, \ldots, x_{h-1}\right) \quad \text { and } \quad \bar{I}_{1}=I_{1} \bar{A} \text {. }
$$

Since ht $I_{1}=h$, it is easily seen that ht $\bar{I}_{1}>0$. This implies that $\overline{0}: \bar{I}_{1}^{\infty}$ is nilpotent in $\bar{A}$. Thus, $\operatorname{dim} \bar{A}=\operatorname{dim} \bar{A} / \overline{0}: \bar{I}_{1}^{\infty}=\operatorname{dim} A /\left[\left(x_{1}, \ldots, x_{h-1}\right): I_{1}^{\infty}\right] \leq q-(h-1)$. But it is clear that $\operatorname{dim} \bar{A}=\operatorname{dim} A /\left(x_{1}, \ldots, x_{h-1}\right) \geq \operatorname{dim} A-(h-1) \geq q-(h-1)$. Hence $\operatorname{dim} A /\left[\left(x_{1}, \ldots, x_{h-1}\right): I_{1}^{\infty}\right]=q-(h-1)$. According to (iv), $x_{1}, \ldots, x_{h-1}$ is an (FC)sequence with respect to $(J, \mathcal{F})$. 
Combining Lemma 3.5 and Proposition 3.6 we get a generalization of [12, Theorem 4.1] which is the main result of this section as follows.

THEOREM 3.7. Let $J$ be an $\mathfrak{m}$-primary ideal of $A$ and let $\mathcal{F}=\left\{I_{n}\right\}_{n \geq 0}$ be a non-nilpotent good filtration of ideals in $A$. Set $q=\operatorname{dim} A / 0: I_{1}^{\infty}, h=\mathrm{ht} I_{1}$, and $\ell=\ell\left(I_{1}\right)$. Let $d_{0}, d_{1}$ be non-negative integers such that $d_{0}+d_{1}=q-1$. Then the following statements hold.

(i) $\quad e_{A}\left(J^{\left[d_{0}+1\right]}, \mathcal{F}^{\left[d_{1}\right]}\right) \neq 0$ if and only if there exists an $(F C)$-sequence $x_{1}, \ldots, x_{d_{1}}$ in $I_{1}$ with respect to $(J, \mathcal{F})$. In this case,

$$
e_{A}\left(J^{\left[d_{0}+1\right]}, \mathcal{F}^{\left[d_{1}\right]}\right)=e\left(J, A /\left[\left(x_{1}, \ldots, x_{d_{1}}\right): I_{1}^{\infty}\right]\right) .
$$

(ii) If $h>0, d_{1}<h$, and $x_{1}, \ldots, x_{d_{1}} \in I_{1}$ is an $(F C)$-sequence with respect to $(J, \mathcal{F})$, then

$$
e_{A}\left(J^{\left[d_{0}+1\right]}, \mathcal{F}^{\left[d_{1}\right]}\right)=e\left(J, A /\left(x_{1}, \ldots, x_{d_{1}}\right)\right) .
$$

(iii) The length of maximal $(F C)$-sequences in $I_{1}$ with respect to $(J, \mathcal{F})$ is an invariant and this invariant is equal to

$$
p=\max \left\{d_{1} \mid e_{A}\left(J^{\left[d_{0}+1\right]}, \mathcal{F}^{\left[d_{1}\right]}\right) \neq 0\right\} .
$$

Moreover, $h-1 \leq p \leq \ell-1$.

Proof. We prove (i). If $e_{A}\left(J^{\left[d_{0}+1\right]}, \mathcal{F}^{\left[d_{1}\right]}\right) \neq 0$ then by induction on $d_{1}$, using Proposition 3.6(i) and (ii), we can easily show the existence of an (FC)-sequence $x_{1}, \ldots, x_{d_{1}}$ in $I_{1}$ with respect to $(J, \mathcal{F})$. Conversely, assume that $x_{1}, \ldots, x_{d_{1}}$ is an (FC)-sequence in $I_{1}$ with respect to $(J, \mathcal{F})$. Set $\bar{A}=A /\left(x_{1}, \ldots, x_{d_{1}}\right), \bar{J}=J \bar{A}$ and $\overline{\mathcal{F}}=\left\{I_{n} \bar{A}\right\}_{n \geq 0}$. Then by Proposition 3.6(ii) we get $e_{A}\left(J^{\left[d_{0}+1\right]}, \mathcal{F}^{\left[d_{1}\right]}\right)=e_{\bar{A}}\left(\bar{J}^{\left[d_{0}+1\right]}, \overline{\mathcal{F}}^{[0]}\right)$. Now by Lemma 3.5(ii),

$$
e_{\bar{A}}\left(\bar{J}^{\left[d_{0}+1\right]}, \overline{\mathcal{F}}^{[0]}\right)=e\left(J, A /\left[\left(x_{1}, \ldots, x_{d_{1}}\right): I_{1}^{\infty}\right]\right) .
$$

Hence

$$
e_{A}\left(J^{\left[d_{0}+1\right]}, \mathcal{F}^{\left[d_{1}\right]}\right)=e\left(J, A /\left[\left(x_{1}, \ldots, x_{d_{1}}\right): I_{1}^{\infty}\right]\right) .
$$

We now prove (ii). Set $\bar{A}=A /\left(x_{1}, \ldots, x_{d_{1}}\right), \bar{J}=J \bar{A}$, and $\overline{\mathcal{F}}=\left\{I_{n} \bar{A}\right\}_{n \geq 0}$. By Proposition 3.6(ii) we easily get

$$
e_{A}\left(J^{\left[d_{0}+1\right]}, \mathcal{F}^{\left[d_{1}\right]}\right)=e_{\bar{A}}\left(\bar{J}^{\left[d_{0}+1\right]}, \overline{\mathcal{F}}^{[0]}\right) .
$$

Since ht $I_{1}=h>d_{1}, \operatorname{ht}\left(I_{1} \bar{A}\right)>0$. Lemma 3.5(ii) now yields

$$
e_{\bar{A}}\left(\bar{J}^{\left[d_{0}+1\right]}, \overline{\mathcal{F}}^{[0]}\right)=e(\bar{J}, \bar{A})=e(J, \bar{A}) .
$$

Hence $e_{A}\left(J^{\left[d_{0}+1\right]}, \mathcal{F}^{\left[d_{1}\right]}\right)=e(J, \bar{A})$.

We now prove (iii). (i) immediately gives the first assertion of (iii) that the length of maximal $(\mathrm{FC})$-sequences in $I_{1}$ with respect to $(J, \mathcal{F})$ is an invariant and this invariant is equal to $p=\max \left\{d_{1} \mid e_{A}\left(J^{\left[d_{0}+1\right]}, \mathcal{F}^{\left[d_{1}\right]}\right) \neq 0\right\}$. By Proposition 3.6(v), $h-1 \leq p$. It remains to show that $p \leq \ell-1$. First, note that $p \leq d_{0}+d_{1}=q-1$. Now assume that $x_{1}, \ldots, x_{p}$ is a maximal (FC)-sequence in $I_{1}$ with respect to $(J, \mathcal{F})$. Set $\bar{A}=A /\left(x_{1}, \ldots, x_{p}\right)$ and $\bar{I}_{1}=I_{1} \bar{A}$. Then by Proposition 3.6(iv), we have

$$
\operatorname{dim} \bar{A} / \overline{0}: \bar{I}_{1}^{\infty}=\operatorname{dim} A /\left[\left(x_{1}, \ldots, x_{p}\right): I_{1}^{\infty}\right]=q-p \geq 1 .
$$


From this it follows that $\bar{I}_{1}$ is non-nilpotent in $\bar{A}$. So by Lemma 3.2, there exists $x \in I_{1}$ such that $x_{1}, \ldots, x_{p}, x$ is a weak-(FC)-sequence with respect to $(J, \mathcal{F})$. Since the length of maximal weak-(FC)-sequences in $I_{1}$ with respect to $(J, \mathcal{F})$ is $\ell$ (by Lemma 3.4), we get $p+1 \leq \ell$, or $p \leq \ell-1$.

Remark 3.8. If we call $\max \left\{d_{1} \mid e_{A}\left(J^{\left[d_{0}+1\right]}, \mathcal{F}^{\left[d_{1}\right]}\right) \neq 0\right\}$ the index of positive definition of mixed multiplicities of $(J, \mathcal{F})$, then Theorem 3.7(iii) shows that this index is equal to the length of maximal (FC)-sequences in $I_{1}$ with respect to $(J, \mathcal{F})$, i.e., $p=\max \left\{d_{1} \mid\right.$ $\left.e_{A}\left(J^{\left[d_{0}+1\right]}, \mathcal{F}^{\left[d_{1}\right]}\right) \neq 0\right\}$. In the case of ideals, this fact was mentioned by Viet [12] in 2000, and Trung [10] in 2001 constructed an example where $p<\ell-1$.

Recall that if $\mathcal{F}$ is an equimultiple filtration then $h t I_{1}=\ell\left(I_{1}\right)$. Hence as an immediate consequence of Theorem 3.7 we obtain the following interesting corollary.

COROLlARY 3.9. Let $J$ be an $\mathfrak{m}$-primary ideal of $A$ and let $\mathcal{F}=\left\{I_{n}\right\}_{n \geq 0}$ be a non-nilpotent good filtration of ideals in A. Suppose that $\mathcal{F}$ is an equimultiple filtration with ht $I_{1}=\ell\left(I_{1}\right)=$ $h>0$. Then the following statements hold.

(i) The length of maximal $(F C)$-sequences in $I_{1}$ with respect to $(J, \mathcal{F})$ is $(h-1)$.

(ii) $e_{A}\left(J^{\left[d_{0}+1\right]}, \mathcal{F}^{\left[d_{1}\right]}\right) \neq 0$ if and only if $d_{1} \leq h-1$.

(iii) If $x_{1}, \ldots, x_{h-1}$ is an $(F C)$-sequence in $I_{1}$ with respect to $(J, \mathcal{F})$, then

$$
e_{A}\left(J^{\left[d_{0}+1\right]}, \mathcal{F}^{\left[d_{1}\right]}\right)=e\left(J, A /\left(x_{1}, \ldots, x_{d_{1}}\right)\right)
$$

for all $d_{1}=0,1, \ldots, h-1$.

In particular, if $I_{1}$ is an $\mathfrak{m}$-primary ideal, then we get the following.

COROLlary 3.10. Let $J, I_{1}$ be $\mathfrak{m}$-primary ideals of $A$ and let $\mathcal{F}=\left\{I_{n}\right\}_{n \geq 0}$ be a good filtration of ideals in $A$. Then there exists an $(F C)$-sequence $x_{1}, \ldots, x_{d-1}$ in $I_{1}$ with respect to $(J, \mathcal{F})$ and we have $e_{A}\left(J^{\left[d_{0}+1\right]}, \mathcal{F}^{\left[d_{1}\right]}\right)=e\left(J, A /\left(x_{1}, \ldots, x_{d_{1}}\right)\right)$ for all $d_{1}=$ $0,1, \ldots, d-1$.

Proof. Since $I_{1}$ is an $\mathfrak{m}$-primary ideal, ht $I_{1}=\ell\left(I_{1}\right)=d$, i.e., $\mathcal{F}$ is an equimultiple filtration. Hence by Corollary 3.9, we obtain the proof.

\section{Some multiplicity formulas for Rees algebras}

In this section, we first extend the multiplicity formula for the Rees algebra of an ideal obtained by Verma [11] to the Rees algebra of a good filtration. Together with the results just established in Section 3, we obtain some multiplicity formulas for Rees algebras of good filtrations.

Let $S$ be a finitely-generated graded algebra over a Noetherian local ring $A$ with the homogeneous maximal ideal $\mathfrak{M}$, and let $M$ be a finitely generated graded $S$-module. We denote by $e(M)$ the Hilbert-Samuel multiplicity $e\left(M_{\mathfrak{M}}\right)$ of the module $M_{\mathfrak{M}}$ over the local ring $S_{\mathfrak{M}}$, and by $S_{+}$the set of elements of positive degrees of $S$. If $I$ is a homogeneous $\mathfrak{M}$-primary ideal of $S$, we shall use the notation $e(I, M)$ instead of $e\left(I S_{\mathfrak{M}}, M_{\mathfrak{M}}\right)$.

Let $\mathcal{F}=\left\{I_{n}\right\}_{n \geq 0}$ be a non-nilpotent good filtration of ideals in $A$. Denote by $R(\mathcal{F})$ the Rees algebra of $\mathcal{F}$ and $R_{j}=\bigoplus_{k \geq j} I_{k} t^{k}$ for any $j \geq 0$. 
LEMMA 4.1. Let $J$ be an $\mathfrak{m}$-primary ideal of $A$ and let $\mathcal{F}=\left\{I_{n}\right\}_{n \geq 0}$ be a non-nilpotent good filtration of ideals in $A$. Set $K=J \oplus\left(\bigoplus_{k \geq 1} I_{k} t^{k}\right)$ and $q=\operatorname{dim} A / 0: I_{1}^{\infty}$. Then $\operatorname{dim} R_{j}=$ $q+1$ and

$$
e\left(K, R_{j}\right)=\sum_{d_{0}+d_{1}=q-1} e_{A}\left(J^{\left[d_{0}+1\right]}, \mathcal{F}^{\left[d_{1}\right]}\right)
$$

for all large $j$.

Proof. Note that $R_{v} \subseteq R_{u}$ and $J^{m} R_{u}+R_{v}=J^{m} I_{u} t^{u} \oplus \cdots \oplus J^{m} I_{v-1} t^{v-1} \oplus R_{v}$ for all $v>u$. Since $\mathcal{F}$ is a good filtration, $I_{u} t^{u} R_{j}=R_{u+j}$ and $R_{u} R_{j}=R_{u+j}$ for all $u \geq 0$ and all large $j$. Hence we can prove by induction on $m$ that

$$
K^{m} R_{j}=J^{m} I_{j} t^{j} \oplus J^{m-1} I_{j+1} t^{j+1} \oplus \cdots \oplus J I_{j+m-1} t^{j+m-1} \oplus R_{m+j}
$$

for all $m \geq 0$ and all large $j$. It follows that

$$
\ell_{A}\left(\frac{K^{m} R_{j}}{K^{m+1} R_{j}}\right)=\sum_{k=0}^{m} \ell_{A}\left(\frac{J^{m-k} I_{j+k}}{J^{m-k+1} I_{j+k}}\right)
$$

for all $m \geq 0$ and all large $j$. By Proposition 2.2, $B(m, n)=\ell_{A}\left(J^{m} I_{n} / J^{m+1} I_{n}\right)$ is the polynomial $P(m, n)$ of degree $q-1$ for all large $m, n$. We may assume that $B(m, n)=$ $P(m, n)$ for all $m, n$ as it does not affect the top degree terms of the polynomial $P(m, n)$. Thus, from (4) we can write

$$
\ell_{A}\left(\frac{K^{m} R_{j}}{K^{m+1} R_{j}}\right)=\sum_{k=0}^{m} \sum_{d_{0}+d_{1}=q-1} e_{A}\left(J^{\left[d_{0}+1\right]}, \mathcal{F}^{\left[d_{1}\right]}\right) \frac{(m-k)^{d_{0}}(j+k)^{d_{1}}}{d_{0} ! d_{1} !}+V(m)
$$

(deg $V(m)<q-1$ ) for all $m \geq 0$ and all large $j$. Now, by using the same argument as in the proof of [11, Theorem 3.1] we get

$$
\ell_{A}\left(\frac{K^{m} R_{j}}{K^{m+1} R_{j}}\right)=\frac{m^{q}}{q !} \sum_{d_{0}+d_{1}=q-1} e_{A}\left(J^{\left[d_{0}+1\right]}, \mathcal{F}^{\left[d_{1}\right]}\right)+W(m)
$$

(deg $W(m)<q$ ) for all $m \geq 0$ and all large $j$. From this it follows that $\operatorname{dim} R_{j}=q+1$ and $e\left(K, R_{j}\right)=\sum_{d_{0}+d_{1}=q-1} e_{A}\left(J^{\left[d_{0}+1\right]}, \mathcal{F}^{\left[d_{1}\right]}\right)$ for all large $j$.

In the following, let $\mathcal{F}=\left\{I_{n}\right\}_{n \geq 0}$ be a good filtration of ideals in $A$ with ht $I_{1}>0$. The following proposition is a generalization of [11, Theorem 3.1].

PROposition 4.2. Let $J$ be an $\mathfrak{m}$-primary ideal of $A$ and let $\mathcal{F}=\left\{I_{n}\right\}_{n \geq 0}$ be a good filtration of ideals in A with ht $I_{1}>0$. Set $K=J \oplus\left(\bigoplus_{k \geq 1} I_{k} t^{k}\right)$. Then

$$
e(K, R(\mathcal{F}))=\sum_{d_{0}+d_{1}=d-1} e_{A}\left(J^{\left[d_{0}+1\right]}, \mathcal{F}^{\left[d_{1}\right]}\right) .
$$

Proof. Since ht $I_{1}>0$, then $0: I_{1}^{\infty}$ is nilpotent. Hence $q=\operatorname{dim} A / 0: I_{1}^{\infty}=\operatorname{dim} A=d$. So by Lemma 4.1 we can choose $j$ large enough such that

$$
e\left(K, R_{j}\right)=\sum_{d_{0}+d_{1}=d-1} e_{A}\left(J^{\left[d_{0}+1\right]}, \mathcal{F}^{\left[d_{1}\right]}\right) .
$$

Put $S=R(\mathcal{F}) / R_{j}$. We first prove that $\operatorname{dim} S=d$. Since $\left[R(\mathcal{F})_{+}\right]^{j} \subseteq R_{j}$, then $S_{+}$is nilpotent. Therefore, every prime ideal of $S$ has the form $P \oplus S_{+}$, where $P$ is a prime ideal of $A$. 
From this it follows that $\operatorname{dim} S=\operatorname{dim} A=d$. Since $\operatorname{dim} S<\operatorname{dim} R_{j}=d+1$, from the exact sequence

$$
0 \longrightarrow R_{j} \longrightarrow R(\mathcal{F}) \longrightarrow S \longrightarrow 0
$$

we get $e(K, R(\mathcal{F}))=e\left(K, R_{j}\right)=\sum_{d_{0}+d_{1}=d-1} e_{A}\left(J^{\left[d_{0}+1\right]}, \mathcal{F}^{\left[d_{1}\right]}\right)$.

As an immediate consequence of this proposition we have the following formula for the multiplicity of Rees algebras of good filtrations.

COROLLARY 4.3. Let $\mathcal{F}=\left\{I_{n}\right\}_{n \geq 0}$ be a good filtration of ideals in A with ht $I_{1}>0$. Then

$$
e(R(\mathcal{F}))=\sum_{d_{0}+d_{1}=d-1} e_{A}\left(\mathfrak{m}^{\left[d_{0}+1\right]}, \mathcal{F}^{\left[d_{1}\right]}\right) .
$$

Remark 4.4. The condition ht $I_{1}>0$ in Proposition 4.2 and Corollary 4.3 can be replaced by a weaker condition: $\operatorname{dim} A / 0: I_{1}^{\infty}=\operatorname{dim} A$. Moreover, from the proof of Lemma 4.1 and Proposition 4.2 we obtain the following interesting corollary.

COROLLARY 4.5. Let $\mathcal{F}=\left\{I_{n}\right\}_{n \geq 0}$ be a non-nilpotent good filtration of ideals in A. Then

$$
\operatorname{dim} R(\mathcal{F})=\max \left\{\operatorname{dim} A, \operatorname{dim}\left[A / 0: I_{1}^{\infty}\right]+1\right\} .
$$

In particular, $\operatorname{dim} R(\mathcal{F})=\operatorname{dim} A+1$ if and only if $\operatorname{dim} A / 0: I_{1}^{\infty}=\operatorname{dim} A$.

Proof. Fix $j$ large enough such that $\operatorname{dim} R_{j}=\operatorname{dim}\left[A / 0: I_{1}^{\infty}\right]+1$ (this is possible by Lemma 4.1). Put $S=R(\mathcal{F}) / R_{j}$. Then from the proof of Proposition 4.2 we have $\operatorname{dim} S=$ $\operatorname{dim} A$. So

$$
\operatorname{dim} R(\mathcal{F})=\max \left\{\operatorname{dim} S, \operatorname{dim} R_{j}\right\}=\max \left\{\operatorname{dim} A, \operatorname{dim}\left[A / 0: I_{1}^{\infty}\right]+1\right\} .
$$

Now from Proposition 4.2, Corollary 4.3 and Theorem 3.7 we get the following extension of [12, Theorem 4.2].

THEOREM 4.6. Let $J$ be an $\mathfrak{m}$-primary ideal of $A$ and let $\mathcal{F}=\left\{I_{n}\right\}_{n \geq 0}$ be a good filtration of ideals in $A$ with ht $I_{1}=h>0$. Assume that $x_{1}, \ldots, x_{p}$ is a maximal $(F C)$-sequence in $I_{1}$ with respect to $(J, \mathcal{F})$, and that $y_{1}, \ldots, y_{p}$ is a maximal $(F C)$-sequence in $I_{1}$ with respect to $(\mathfrak{m}, \mathcal{F})$. Set $K=J \oplus\left(\bigoplus_{k \geq 1} I_{k} t^{k}\right)$. Then:

(i) $e(K, R(\mathcal{F}))=\sum_{i=0}^{h-1} e\left(J, A /\left(x_{1}, \ldots, x_{i}\right)\right)+\sum_{i=h}^{p} e\left(J, A /\left[\left(x_{1}, \ldots, x_{i}\right): I_{1}^{\infty}\right]\right)$;

(ii) $e(R(\mathcal{F}))=\sum_{i=0}^{h-1} e\left(A /\left(y_{1}, \ldots, y_{i}\right)\right)+\sum_{i=h}^{p} e\left(A /\left[\left(y_{1}, \ldots, y_{i}\right): I_{1}^{\infty}\right]\right)$.

In the case when $\mathcal{F}$ is an equimultiple good filtration, we obtain the following result by combining Proposition 4.2 and Corollary 4.3 with Corollary 3.9. This is a generalization of [13, Theorem 3.2].

COROLLARY 4.7. Let $J$ be an $\mathfrak{m}$-primary ideal of $A$ and let $\mathcal{F}=\left\{I_{n}\right\}_{n \geq 0}$ be an equimultiple good filtration of ideals in A with ht $I_{1}=\ell\left(I_{1}\right)=h>0$. Assume that $x_{1}, \ldots, x_{h-1}$ is a maximal $(F C)$-sequence in $I_{1}$ with respect to $(J, \mathcal{F})$, and that $y_{1}, \ldots, y_{h-1}$ is a maximal $(F C)$-sequence in $I_{1}$ with respect to $(\mathfrak{m}, \mathcal{F})$. Set $K=J \oplus\left(\bigoplus_{k \geq 1} I_{k} t^{k}\right)$. Then:

(i) $e(K, R(\mathcal{F}))=\sum_{i=0}^{h-1} e\left(J, A /\left(x_{1}, \ldots, x_{i}\right)\right)$;

(ii) $e(R(\mathcal{F}))=\sum_{i=0}^{h-1} e\left(A /\left(y_{1}, \ldots, y_{i}\right)\right)$. 
Finally, if $I_{1}$ is an m-primary ideal, then as an immediate consequence of Proposition 4.2, Corollary 4.3 and Corollary 3.10, we have the following generalization of [12, Theorem 4.3] to the case of good filtrations.

COROLlary 4.8. Let $J, I_{1}$ be m-primary ideals of $A$ and let $\mathcal{F}=\left\{I_{n}\right\}_{n \geq 0}$ be a good filtration of ideals in A. Assume that $x_{1}, \ldots, x_{d-1}$ is a maximal $(F C)$-sequence in $I_{1}$ with respect to $(J, \mathcal{F})$, and that $y_{1}, \ldots, y_{d-1}$ is a maximal $(F C)$-sequence in $I_{1}$ with respect to $(\mathfrak{m}, \mathcal{F})$. Set $K=J \bigoplus\left(\bigoplus_{k \geq 1} I_{k} t^{k}\right)$. Then:

$$
\begin{aligned}
& \text { (i) } \quad e(K, R(\mathcal{F}))=\sum_{i=0}^{d-1} e\left(J, A /\left(x_{1}, \ldots, x_{i}\right)\right) \text {; } \\
& \text { (ii) } e(R(\mathcal{F}))=\sum_{i=0}^{d-1} e\left(A /\left(y_{1}, \ldots, y_{i}\right)\right) .
\end{aligned}
$$

Acknowledgement. This research was in part supported by a grant from NAFOSTED.

\section{REFERENCES}

[1] P. B. Bhattacharya. The Hilbert function of two ideals. Proc. Cambridge Philos. Soc. 53 (1957), 568-575.

[2] C. Huneke and I. Swanson. Integral Closure of Ideals, Rings, and Modules (London Mathematical Lecture Note Series, 336). Cambridge University Press, 2006.

[3] D. Katz and J. Verma. Extended Rees algebras and mixed multiplicities. Math. Z. 202 (1989), 111-128.

[4] N. T. Manh and D. Q. Viet. Mixed multiplicities of modules over Noetherian local rings. Tokyo J. Math. 29(2) (2006), 325-345.

[5] D. G. Northcott and D. Rees. Reductions of ideal in local rings. Proc. Cambridge Philos. Soc. 50 (1954), $145-158$.

[6] L. J. Ratliff and D. Rush. Two notes on reductions of ideals. Indiana Univ. Math. J. 27 (1978), 929-934.

[7] D. Rees. A note on analytically unramified local rings. J. London Math. Soc. 36 (1961), 24-28.

[8] D. Rees. Generalizations of reductions and mixed multiplicities, J. London Math. Soc. 29 (1984), $397-414$.

[9] B. Teissier. Cycles èvanescents, sections planes, et conditions de Whitney. Singularités à Cargése 1972, Astérisque 7-8 (1973), 285-362.

[10] N. V. Trung. Positivity of mixed multiplicities. Math. Ann. 319 (2001), 33-63.

[11] J. K. Verma. Rees algebras and mixed multiplicities. Proc. Amer. Math. Soc. 104 (1988), 1036-1044.

[12] D. Q. Viet. Mixed multiplicities of arbitrary ideals in local rings. Comm. Algebra 28(8) (2000), 3803-3821.

[13] D. Q. Viet. On some properties of (FC)-sequences of ideals in local rings. Proc. Amer. Math. Soc. 131 (2003), 45-53.

[14] D. Q. Viet. Sequences determining mixed multiplicities and reductions of ideals. Comm. Algebra 31(10) (2003), 5047-5069.

[15] D. Q. Viet. Reductions and mixed multiplicities of ideals. Comm. Algebra 32(11) (2004), 4159-4178.

[16] D. Q. Viet and T. T. H. Thanh. Multiplicity and Cohen-Macaulayness of fiber cones of good filtrations. Kyushu J. Math. 65(1) (2011), 1-13.

\section{Duong Quoc Viet}

Department of Mathematics

Hanoi National University of Education

136 Xuan Thuy street

Hanoi, Vietnam

(E-mail:duongquocviet@fmail.vnn.vn)
Le Van Dinh

Department of Mathematics

Hanoi National University of Education

136 Xuan Thuy street

Hanoi, Vietnam

(E-mail:dinhlevands@gmail.com) 